\title{
Photopolymerization of electroactive film applied to full polymer electrochromic device
}

\author{
P. Y. Chang, C. H. Yang* \\ Department of Chemical and Materials Engineering National University of Kaohsiung, 811 Kaohsiung, Taiwan
}

Received 11 August 2016; accepted in revised form 19 October 2016

\begin{abstract}
Electroactive films of thiophene-acrylate polymers were simultaneously photopolymerized by means of ultraviolet (UV) irradiation using diphenyliodonium hexafluorophosphate and acrylate special photoinitiators (PIs) as a mixed photoinitiator. Free radicals from PIs can promote cationic polymerization of thiophenes. Electrical conductivity and transmittance of the electroactive film are high to $10^{-2} \mathrm{~S} \cdot \mathrm{cm}^{-1}$ and $>90 \%$. Electroactivity of the photopolymerized polymer film was confirmed by electro-polymerization of aniline on this film in aqueous solution and employed to assemble a full polymer electrochromic device having a superior optical contrast of $36.6 \%$.
\end{abstract}

Keywords: polymer blends and alloys, thiophene-acrylate polymer composites, electroactive film, photopolymerization, electrochromic device

\section{Introduction}

Conducting polymers are promising materials for applications in molecular electronics, molecular wires, and devices [1,2], which combine chemical and electrochemical properties of polymers with electronic properties of metals and semiconductors. In the heterocyclic conjugated polymers, polythiophenes have attracted special attention because of their electrical characteristics, environmental stability in doped and undoped states, and reversible redox switching [3], which contain a $\pi$-conjugated carbon backbone and exhibit particular electrochemical [3], electrochromic [4] and electromagnetic shielding [5] characteristics. These materials provide a basis for some new technologies [6, 7]. Polymerization of thiophenes is usually achieved by chemical [7-9], electrochemical [3], and photochemical $[6,10]$ processes, forming the corresponding polythiophenes with diverse morphology coupled with different physical and chemical properties. However, the solubility of polythiophenes is very low in most organic solvents, resulting in poor processability, limiting their practical application. On the other hand, polyaniline (PAN) is the simplest linear conjugated macromolecule and a representative of conducting polymers [11]. To combine two or more desirable properties, multicomposites can provide additional stability for molecular assembly. The functionality of a fine device results from a combination of physical and chemical processes (e.g., electron and energy transfer). Such a device requires controlling molecular orientation and organization on the nanoscale, because its function significantly depends on the local chemical environment. Therefore, scientists greatly desire to develop methods for the controllable assembly of multicomponent nanostructures.

Photopolymerization has recently been proposed for the fabrication of solar cells [12, 13], electrochromic and photoelectrochromic devices [14, 15]. Photopolymerization reactions have been developed for many years $[16,17]$, which involve a polymerizable radical or cationic matrix. Free radical photopoly-

"Corresponding author, e-mail: yangch@nuk.edu.tw

C BME-PT 
merization (FRP) is used to polymerize unsaturated monomers such as acrylates, whereas cationic photopolymerization $(\mathrm{CP})$ is used to polymerize heterocyclic monomers, e.g. thiophenes. A photoinitiator (PI) has to be advisably selected to absorb the irradiation wavelengths $[16,17]$. In FRP, PIs are usually designed to absorb the near UV/visible wavelengths. Upon irradiation, PI becomes excited (PI*) and generates a radical, $\mathrm{R} \cdot$, either directly through cleavage or in the presence of an electron/hydrogen donor. Then, a radical attacks a monomer $(\mathrm{M})$ to form a radical monomer (RM•), an active species, which can consecutively contacts other monomers forming the propagation of molecular chains. Finally, two propagating chains combine to produce a polymer as shown in Equations (1) and (2):

$$
\mathrm{PI}(\mathbf{h v}) \rightarrow \mathrm{PI}^{*} \rightarrow \mathrm{R} \bullet
$$

$\mathrm{R} \bullet+\mathrm{n} \mathrm{M}$ (radical monomer) $\rightarrow \mathrm{RM}_{\mathrm{n}} \bullet$ (propagation)

$$
\rightarrow \text { polymer }
$$

In $\mathrm{CP}$, the PIs mainly absorb in the range of UV wavelengths. Onium salts (e.g., iodonium salt) are generally used as PI [4]. Homolytic/heterolytic decomposition followed by hydrogen transfer reactions leads to a proton (see Equations (3)-(5)):

$$
\mathrm{PI}\left(\mathrm{Ph}_{2} \mathrm{I}^{+} \mathrm{X}^{-}\right)(\mathbf{h v}) \rightarrow \mathrm{PI}^{*} \rightarrow \mathrm{PhI}^{+\bullet} \mathrm{X}^{-}+\mathrm{Ph} \bullet
$$

$\mathrm{PhI}^{+\bullet} \mathrm{X}^{-}+\mathrm{M}-\mathrm{H}$ (cationic monomer) $\rightarrow$

$\mathrm{H}^{+} \mathrm{X}^{-}+\mathrm{PhI}+\mathrm{M} \bullet$

$\mathrm{M} \bullet+\mathrm{M} \bullet \rightarrow \mathrm{M}-\mathrm{M} \rightarrow \rightarrow \rightarrow$ polymer

were $\mathrm{X}^{-}: \mathrm{PF}_{6}^{-}, \mathrm{M}-\mathrm{H}$ : thiopene monomers, e.g. 3,4ethylenedioxythiophene (EDOT) or thiophene-3carboxylic acid (TCA) in this work.

Following reactions 3 and 4, hydrogen abstraction reactions formed both radical cations $\left(\mathrm{PhI}^{+}\right)$and protonic acids $\left(\mathrm{H}^{+}\right)$which are greatly active towards monomers and can directly initiate polymerization. To construct an electrochromic device, it is necessary to compensate the coulombic change used for the redox (i.e., coloring/bleaching) process of a complementary modified electrode [18]. The optical contrast of the device is promoted if this complementary electrode system also has complementary electrochromic color changes. Therefore, poly(3,4-ethylenedioxythiophene)/poly(styrene sulfonate) (PEDOT/ PSS) was chosen as the complementary electro- chromic material to attain this effect. PEDOT exhibits an excellent stability to air exposure even at high temperature [19]. PEDOT is presently used for charge dissipation coating [20] and in electrochromic devices because of its significantly transparent oxidized state and opaque, deep blue, reduced state [21]. Furthermore, PEDOT also shows a high conductivity $\left(200 \mathrm{~S} \cdot \mathrm{cm}^{-1}\right)$ [22]. Generally, indium tin oxide (ITO) has been employed as the substrate in electrochromic devices [20,21]. To date, there is lack of the use of real polymer substrates in assembling a full polymer electrochromic device.

Thiophenes, EDOT and TCA, were blend into acrylate monomer forming a mixture of different monomer system. These mixed monomers were in-situ photoinitiated by diphenyliodonium hexafluorophosphate $\left(\mathrm{Ph}_{2} \mathrm{I}^{+} \mathrm{PF}_{6}{ }^{-}\right)$and acrylate special photoinitiators (for example, diphenyl (2, 4, 6-trimethylbenzoyl)phosphine oxide, 1-hydroxy-cyclohexyl-phenyl-ketone, etc.). This polymerization reaction is designed to use UV-light irradiation to obtain an electroactive composite film. Here, the carboxylic acid on TCA was designed to act as a self-dopant in the conductive polythiophene chains, forming a $\mathrm{P}$ (EDOT-co-TCA)/ polyacrylate composite film. Then, polyaniline film can be electrodeposited on this electroactive composite polymer film achieving an electrochromic electrode. It was desirable to create a simple approach that would form a uniform nanoarchitecture film, whereas the fabrication was expected to be independent of the nature, size, and topology of the substrate. The charged molecules (such as propagating molecule chains) seemed to be a good candidate for film buildup on this electroactive polymer composite substrate, because it possesses the least steric requirement of all chemical bonds. In fact, it has rarely been studied with respect to the film formation of propagating conducting polymers on such a transparent electroactive polymer composite film. The formation of polyaniline thin films has important consequences on this composite film and is therefore of interest in large-scale processes such as electrode materials in a full polymer electrochromic device. However, this process is developed to fabricate a uniform thin film of electroactive substrates in further application. These applications may benefit from a better understanding of electroactive composite films as a model electrode substrate, as these can be well characterized by a wide variety of physical techniques. A series of ultrathin films was visually 
observed during these processes. These films were integrated into a full polymer electrochromic device with a superior performance.

\section{Experimental}

\subsection{General information}

Aniline (AN, Aldrich, Milwaukee, USA) was distilled under reduced pressure. Lithium perchlorate $\left(\mathrm{LiClO}_{4}\right.$, Aldrich, Milwaukee, USA), 3,4-ethylenedioxythiophene (EDOT, Aldrich, Milwaukee, USA), thiophene-3-carboxylic acid (TCA, Aldrich, Milwaukee, USA), aniline (AN, Aldrich, Milwaukee, USA), ethoxylated bisphenol A diacrylate (SR601NS, Sartomer, Exton, USA), diphenyliodonium hexafluorophosphate (Ph2I+PF6-, Aldrich, Milwaukee, USA), diphenyl (2, 4, 6-trimethylbenzoyl)-phosphine oxide (TPO, Ciba, Basel, Switzerland), 1-hydroxy-cyclohexyl-phenyl-ketone (Irgacure 184, Ciba, Basel, Switzerland), 2-methyl-1-[4-(methylthio) phenyl]-2-(4-morpholinyl)-1-propanone (Irgacure 907, Ciba, Basel, Switzerland), polyethylene oxide (PEO, MW 400000, Fluka, Tokyo, Japan), propylene carbonate (PC, Fluka, Tokyo, Japan), and dimethylformamide (DMF, Aldrich, Milwaukee, USA) were used as received.

\subsection{Fabrication of electroactive composite film}

A typical photopolymerization was performed as follows: $1.44 \mathrm{~g}$ of TCA and $2.88 \mathrm{~g}$ of $\mathrm{Ph}_{2} \mathrm{I}^{+} \mathrm{PF}_{6}{ }^{-}$photoinitiator dissolved in $14.4 \mathrm{~mL}$ of DMF and mixed with 2.88-8.64 g EDOT denoted as thiophene solution. Multiple photoinitiators ( $0.45 \mathrm{~g}$ of TPO, $0.45 \mathrm{~g}$ of Irgacure 184, and $0.1 \mathrm{~g}$ of Irgacure 907 ) were dissolved in $1 \mathrm{~mL}$ of acetone and mixed with $4.5 \mathrm{~g}$ of ethoxylated bisphenol A diacrylate (SR-601NS) denoted as acrylate solution. Then, the former solution was thoroughly mixed with the latter solution yielding a homogeneous mixture. Several drops of the above mixed solution were dropped on the surface of polyimide substrate $\left(1 \times 1 \mathrm{~cm}^{2}\right)$ using a pipette, and the film was formed by using spin-coating with the consecutive steps of $2000 \mathrm{rpm}$ for $20 \mathrm{~s}$ and $3000 \mathrm{rpm}$ for $10 \mathrm{~s}$. This thin film was exposed to ultraviolet light irradiation of $125 \mathrm{~W}$ (emission peak at $365 \mathrm{~nm}$, Ausbond, Shanghai, China) keeping the distance of $25 \mathrm{~cm}$ from the film to mercury bulb for $10 \mathrm{~min}$, the power level was $8 \mathrm{~mW} / \mathrm{cm}^{2}$ measured at $365 \mathrm{~nm}$, performing photopolymerization to generate a pale dark coating on the polyimide substrate. The residual solvents in the resulting coating were removed at $80^{\circ} \mathrm{C}$ for $6 \mathrm{~h}$ in an oven. Finally, this electroactive film was carefully peeled off the substrate.

\subsection{Electropolymerization of aniline on electroactive composite film}

Electrochemical polymerization using cyclic voltammety was performed on a PGSTAT30 electrochemical Analyzer (AUTOLAB Electrochemical Instruments, Amsterdam, Netherlands). A three-electrode cell assembly was utilized with $\mathrm{Ag} / \mathrm{AgCl}$ electrode as reference. Platinum wire was used as counter electrode. An electroactive composite film of area $1 \mathrm{~cm}^{2}$ was used as working electrode. A Luggin capillary, whose tip was set at a distance of about $1 \mathrm{~mm}$ from the surface of the working electrode, was used to minimize errors due to $i \mathrm{R}$ drop in the electrolytes. Electropolymerization was achieved by cycling the potential in the range of -200 to $1000 \mathrm{mV}$ (vs. $\mathrm{Ag} / \mathrm{AgCl}$ ) with a sweep rate of $50 \mathrm{mV} \cdot \mathrm{s}^{-1}$ in $0.1 \mathrm{~N}$ AN containing $1 \mathrm{~N} \mathrm{HClO}_{4}$ aqueous solution under nitrogen atmosphere and the cyclic voltammograms (CVs) were recorded simultaneously with reaction. An electrochromic electrode of PAN/P(EDOT-co-TCA)/polyacrylate was obtained via the above electropolymerization.

\subsection{Assembly of electrochromic devices}

Gel-type electrolyte was first prepared as follows: $0.1 \mathrm{M} \mathrm{LiClO}_{4}$ and $1 \mathrm{wt} \%$ PEO were dissolved in PC to form a gel-type electrolyte. A PAN | P(EDOT-coTCA)/polyacrylate film, a bare P(EDOT-co-TCA)/ polyacrylate film, and the gel-type electrolyte were sandwiched to assemble an electrochromic device structure: $\mathrm{P}(\mathrm{EDOT}-c o$-TCA)/polyacrylate $(\sim 8 \mu \mathrm{m}) \|$ $\mathrm{LiClO}_{4}$-PEO-PC $(\sim 20 \mu \mathrm{m}) \|$ PAN $(\sim 100 \mathrm{~nm}) \mid$ $\mathrm{P}($ EDOT-co-TCA $) /$ polyacrylate $(\sim 8 \mu \mathrm{m})$. The electrodes were carefully pressed against each other to obtain good adhesion (polymer electrolyte thickness $\sim 20 \mu \mathrm{m})$.

\subsection{Measurements}

Infrared spectra were recorded on a Perkin-Elmer RXI FT-IR spectrometer. Absorption spectra were measured using a Perkin-Elmer (Lambda 25, Waltham, USA) UV-visible spectrophotometer. The microstructure of the sample was investigated by scanning electron microscopy (SEM, Hitachi, S-4800, Tokyo, Japan). Material thermal stability was measured by the differential scanning calorimetry (DSC, SDT-Q600, TA Instruments, New Castle, USA). Im- 
pedance measurements were performed using thin films prepared previously of $1 \mathrm{~cm}^{2}$ in area. The conductivity of the conductive composite films sandwiched between two stainless steel electrodes was obtained on a PGSTAT30 Electrochemical analyzer under an oscillation potential of $10 \mathrm{mV}$ from $100 \mathrm{kHz}$ to $0.1 \mathrm{~Hz}$. Electrochromic device was connected in a two-electrode configuration to a PGSTAT30 electrochemical analyzer. The potentials are reported here with respect to the $\mathrm{P}(\mathrm{EDOT}-$-co-TCA)/polyacrylate electrode. The entire cell structure $\left(1 \mathrm{~cm}^{2}\right.$ surface area) was sealed with epoxy resin. Double potential chronoamperometry was done with the assembled cell by use of a PGSTAT 30 electrochemical analyzer. In situ optical characterization was made using a Perkin-Elmer (Lambda 25) UV-visible spectrophotometer in the UV-visible region.

\section{Results and discussion}

\subsection{Charateristics of}

\section{P(EDOT-co-TCA)/polyacrylate substrates}

The absorption spectrum of the mixture of thiophene dreivatives and $\mathrm{Ph}_{2} \mathrm{I}^{+} \mathrm{PF}_{6}{ }^{-}$in acrylate medium would not change in dark over one week, indicating that the charge transfer complex forms in the ground state but the electron-transfer reactions do not happen between ground-state onium salt and thiophenes. This suggests that polymers cannot be formed in the dark environment. The film of acrylate monomer mixed acrylate special PIs (Darocur TPO + Irgacure 184 + Irgacure 907), irradiated at the light where acrylate special PIs absorb the corresponding wavelength of $365 \mathrm{~nm}$, becomes a transparent well-cured film in $2 \mathrm{~min}$. In contrast, thin-film of thiophene mixture (TCA and EDOT) alone becomes dark color taking 15 min at irradiation of the same wavelength. Furthermore, the cast film of the mixed monomer mixture was irradiated at the light where diphenyliodonium salt and acrylate special PIs simultaneously absorb the wavelength of $365 \mathrm{~nm}$, this film gradually becomes dark and harder without sticking hands (see later in Figure 3) within 5 min, implying that the free radicals from acrylate special PIs are able to significantly promote cationic polymerization of thiophenes. A possible mechanism of the dual photopolymerization is described as follows. In this free radical promoted cationic polymerization (FRPCP), a radical, $\mathrm{R} \cdot$, is formed from a radical source of a PI and then oxidized by $\mathrm{Ph}_{2} \mathrm{I}^{+}$to generate $\mathrm{Ph}_{2} \mathrm{I} \bullet$ and a cation, $\mathrm{R}^{+}$, which would improve the cationic polymeriza- tion of heterocyclic monomers (thiophenes). Note that the $\mathrm{Ph}_{2} \mathrm{I} \bullet$ species are easily decomposed into $\mathrm{PhI}$ and $\mathrm{Ph} \cdot[4,9,10]$ (see Equations (6)-(8)):

$\mathrm{PI}(\mathrm{hv}) \rightarrow \mathrm{PI}^{*} \rightarrow \mathrm{R} \cdot$

$\mathrm{R} \bullet+\mathrm{Ph}_{2} \mathrm{I}^{+} \rightarrow \mathrm{R}^{+}+\mathrm{Ph}_{2} \mathrm{I} \bullet\left(\rightarrow \mathrm{R}^{+}+\mathrm{PhI}+\mathrm{Ph} \bullet\right)$

$\mathrm{R}^{+}+\mathrm{M}-\mathrm{H}$ (cationic monomer) $\rightarrow \longrightarrow \rightarrow$ polymer

In practice, FRPCP is used to perform a cationic polymerization under exposure at $>350 \mathrm{~nm}$ (up to $700 \mathrm{~nm}$ ) and inhibit the oxygen quenching of the radicals. In cationic photopolymerization TCA-EDOT, the generated radical cations $\left(\mathrm{PhI}^{+\bullet}\right)$ from photolysis of the iodonium salt and cations $\left(\mathrm{R}^{+}\right)$produced from a radical source of PIs, are simultaneously responsible for polymerization of thiophenes in the film. The radical cation transfers single electron to thiophene forming thiophene radical cation. A radical source of a PI transfers a positive charge of a cation $\left(\mathrm{R}^{+}\right)$to sulfur atom on thiophene yielding a thiophene radical cation, whereas $\mathrm{R}^{+}$is then reduced to $\mathrm{R} \bullet$. The second step combines two radical cations to form a dihydrodimer dication which produces a dimer through loss of two protons and re-aromatization $[4,9,10]$. Because the dimer yields an active radical cation which attaches monomer to perform chain extension, the photoinduced electron transfer and chemical coupling consecutively perform until the formation of black conductive polymer, shown in Equation (9), generating the polymer film. At this stage, the light incident is obstructed leading to a standstill polymerization. When the above reactions are in progress, a radical $(\mathrm{R} \bullet$ ) produced from a radical source of PIs also can simultaneously photoinitiate polymerization of acrylate matrix in the film. Consequently, irradiation of the cast blend film becomes a dry film in $5 \mathrm{~min}$.

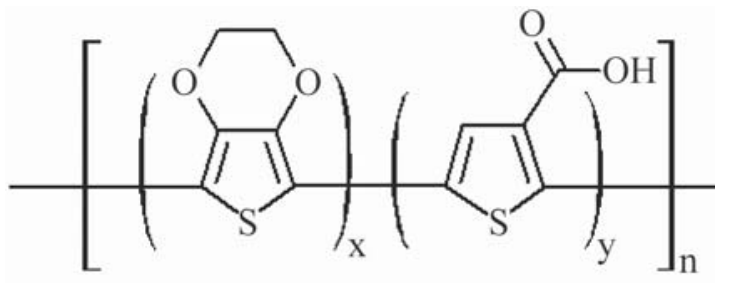

In this work, a difunctional acrylate monomer of ethoxylated bisphenol A diacrylate was photopolymerized into a crosslinked network blended with polythiophenes. The chemical structure of the network blended matrix was analyzed by using FT-IR rather than NMR because the matrix cannot be completely 


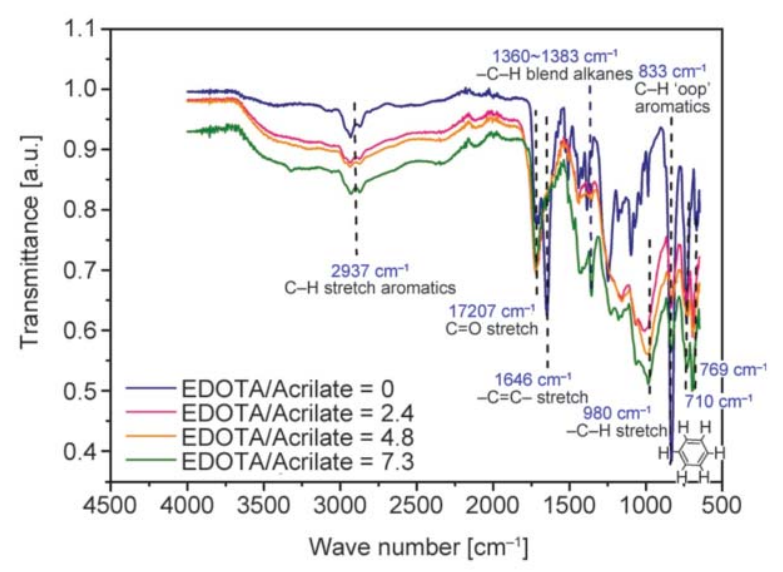

Figure 1. FT-IR spectra of poly(EDOT-co-TCA)-polyacrylate blend films varying the EDOT/acrylate ratio

dissolved in organic solvents. FT-IR measurements (Figure 1) of the photopolymerized products showed $-\mathrm{C}=\mathrm{C}$ stretch at $1646 \mathrm{~cm}^{-1}, \mathrm{C}-\mathrm{H}$ stretch of aromatics at $2937 \mathrm{~cm}^{-1}, \mathrm{C}-\mathrm{H}$ out of plane of aromatics at $833 \mathrm{~cm}^{-1},=\mathrm{C}-\mathrm{H}$ bend at $980 \mathrm{~cm}^{-1}, \mathrm{C}=\mathrm{O}$ stretch at $1720 \mathrm{~cm}^{-1}$. These absorption bands are specific to polythiones (poly(EDOT-co-TCA)). Results clearly confirm that TCA and EDOT units bonded in the resulting conductive polythiophene chains under the photopolymerization conditions. $\mathrm{C}=\mathrm{O}$ stretch at $1720 \mathrm{~cm}^{-1}, \mathrm{C}-\mathrm{H}$ bend of alkanes at $1360-1383 \mathrm{~cm}^{-1}$ coupled with the above aromatic charateristic bands exhibited that polyacrylate was also successfully incorporated to form polymer.

The UV-vis spectra of poly(EDOT-co-TCA)/polyacrylate blend films display absorption maxima at 375 and $650 \mathrm{~nm}$ in Figure 2, indicating that the conductive polymer probably exists as a mixed forms with different level of conjugation. The transmittance of pure polyacrylate is high (ca. 100\%), but this value decreases with increasing the mole ratio of

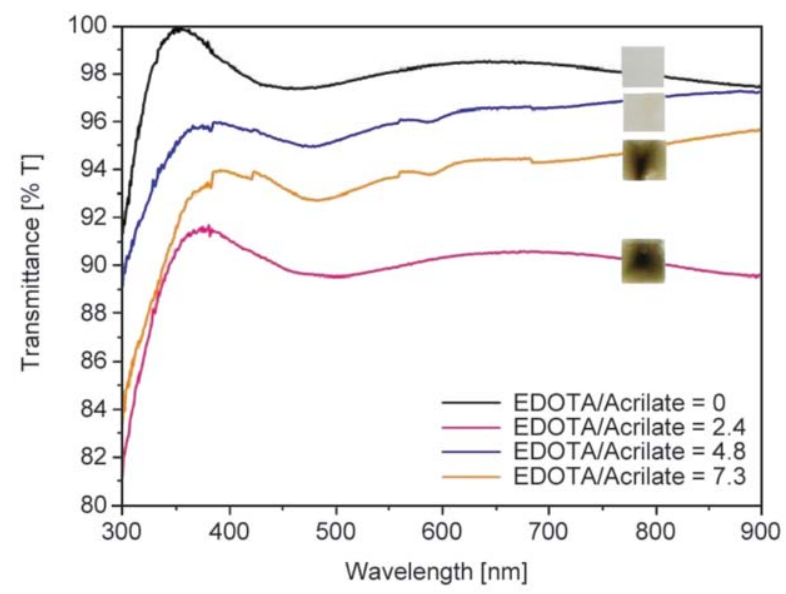

Figure 2. UV-vis spectra of poly(EDOT-co-TCA)-polyacrylate blend films varying the EDOT/acrylate ratio
EDOT/acrylate, indicating that TCA and EDOT units are polymerized to yield a polymer homogeneously mixed in the polyacrylate matrix.

Figure 3 shows SEM morphology on these conductive composites. A flat surface was observed on the polyacrylate film (Figure 3a) and some granular structures on poly(EDOT-co-TCA)/polyacrylate blend films (Figures 3b-3d). The size of granules decreases with increasing the ratio of EDOT/acrylate as shown in Figure 3e. Energy dispersive spectroscopy (EDS) analysis gave a lower level of S composition on the top surface of spheres as compared that of the flat positions on the entire film (Figure 3f). This result arises from the density difference between thiophenes $\left(1.33 \mathrm{~g} / \mathrm{mL}\right.$ at $\left.25^{\circ} \mathrm{C}\right)$ and acrylate $(1.15 \mathrm{~g} / \mathrm{mL}$ at $25^{\circ} \mathrm{C}$ ) as well as the polarity difference in individual polymer materials.

Impedance measurements were performed using thin films prepared previously of about $8 \mu \mathrm{m}$ in thickness and $1 \mathrm{~cm}^{2}$ in area. The conductivity of the conductive composite films sandwiched between two stainless steel electrodes was obtained on a PGSTAT30 Electrochemical analyzer (AUTOLAB Electrochemical Instrument, The Netherlands) under an oscillation potential of $10 \mathrm{mV}$ from $100 \mathrm{kHz}$ to $0.1 \mathrm{~Hz}$. Figure $4 \mathrm{a}$ illustrates the temperature dependence of conductivity for the conductive composite films, indicating that the conductivity increases with increasing EDOT/ acrylate ratio and also increasing temperature. The temperature effect is not particularly significant at relatively lower EDOT/acrylate; there exists a jump increase in the conductivity with high EDOT/acrylate ratio of 7.3 in the range of $70-80^{\circ} \mathrm{C}$, exactly corresponding to the glass transition temperature $\left(T_{\mathrm{g}}\right)$ of polyacrylate matrix (Figure $4 \mathrm{~b}$ ) which is beneficial to the conjugation of polythiophene chains.

Cyclic voltammetry was employed to test the electroactivity of these conductive composite films on ITO substrates. Cyclic voltammograms (CVs) of these films in an acetonitrile/AcOH $(7 / 1, \mathrm{v} / \mathrm{v})$ solution containing $0.1 \mathrm{M}$ tetrabutylammonium tetrafluoroborate $\left(\mathrm{TBABF}_{4}\right)$ and $0.1 \mathrm{M}$ ferrocene are shown in Figure 5. One redox couple can be observed in the conductive films, corresponding to the ferrocene/ferrocenium ion $\left(\mathrm{Fc} / \mathrm{Fc}^{+}\right)$redox wave. The peak current is proportional to the mole ratio of EDOT/acrylate in these composite films. This results from the increase of surface area (Figure 3 ) and active sites on the conductive films with increasing EDOT/acrylate ratio. This result is similar to that of electrical con- 


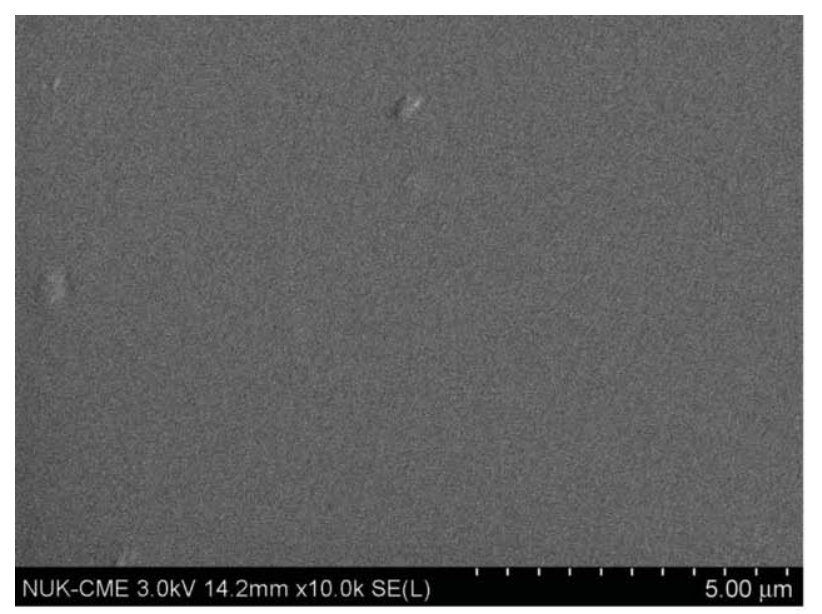

a)

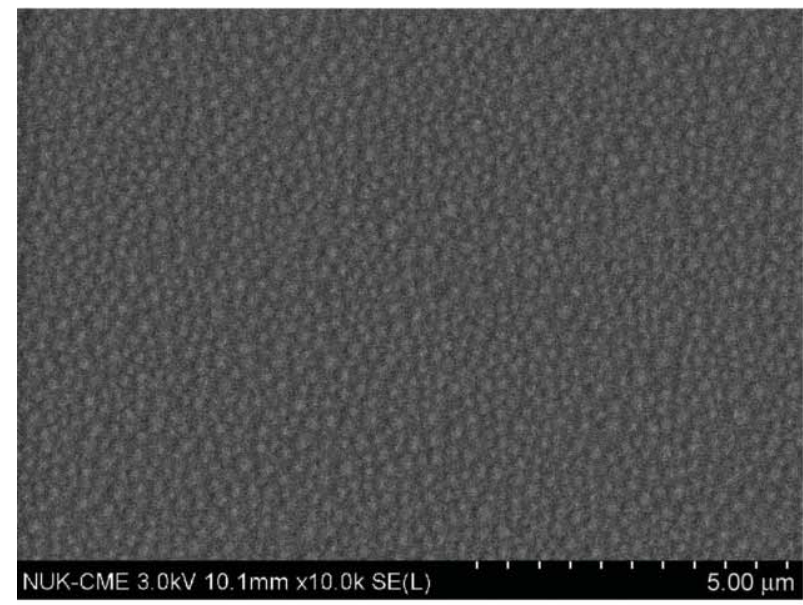

c)

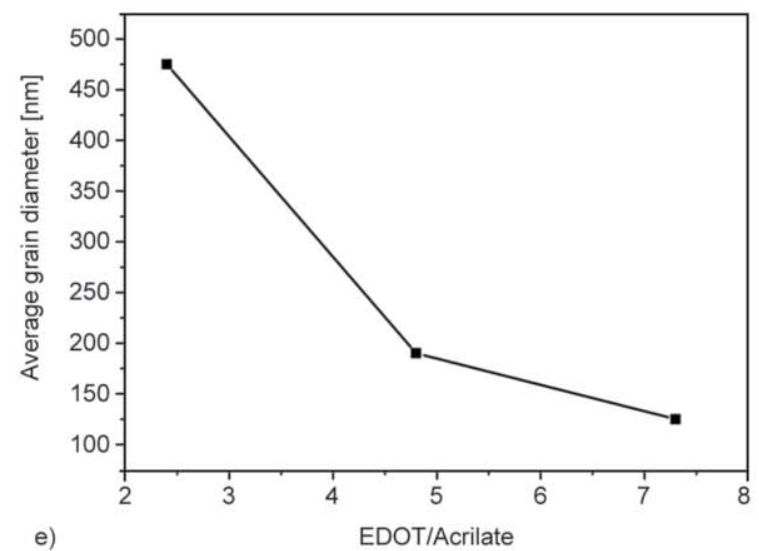

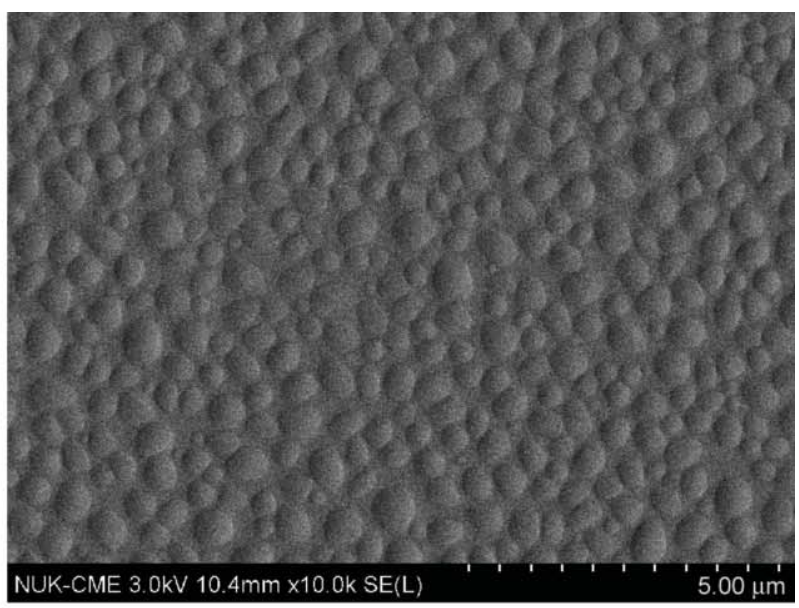

b)

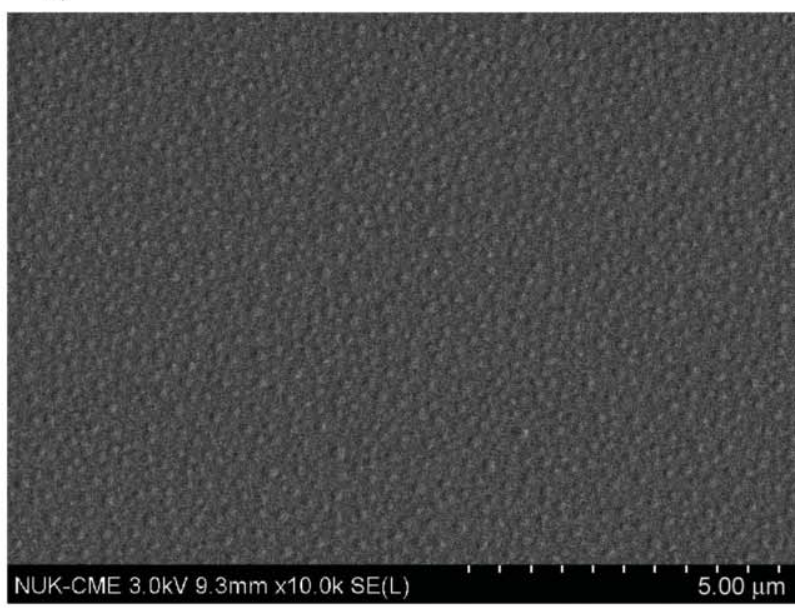

d)

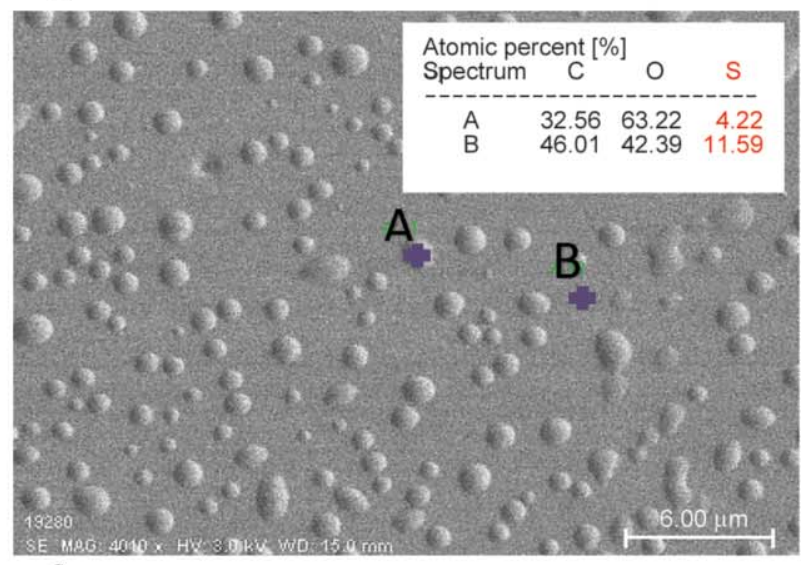

f)

Figure 3. SEM micrographs of poly(EDOT-co-TCA)-polyacrylate blend films varying the EDOT/acrylate ratio: (a) 0 , (b) 2.4 , (c) 4.8 , and (d) 7.3 ; (e) dependence of grain diameter on the films against EDOT/acrylate ratio; (f) EDS elemental analysis on the film with EDOT/acrylate ratio of 2.4

ductivity of these P(EDOT-co-TCA)/polyacrylate films. It is worth mentioning that the $\mathrm{CV}$ of ferrocene recorded on the poly(EDOT-co-TCA)/polyacrylate blend electrodes is not typical with an irreversible redox wave. The oxidative wave is much greater than the reductive one. One possible explanation for this result is that the reductive ability of poly(EDOT-coTCA) would be inhibited by the blended polymer of polyacrylate. Because there existed inhibition effect of the reductive ability, the concentration of ferrocene was added high to $0.1 \mathrm{M}$ to detect redox behavior. However, the poly(EDOT-co-TCA)/polyacrylate blend can still be employed as an electrode substrate for electrodepositing polyaniline because of its electrical conductivity. 

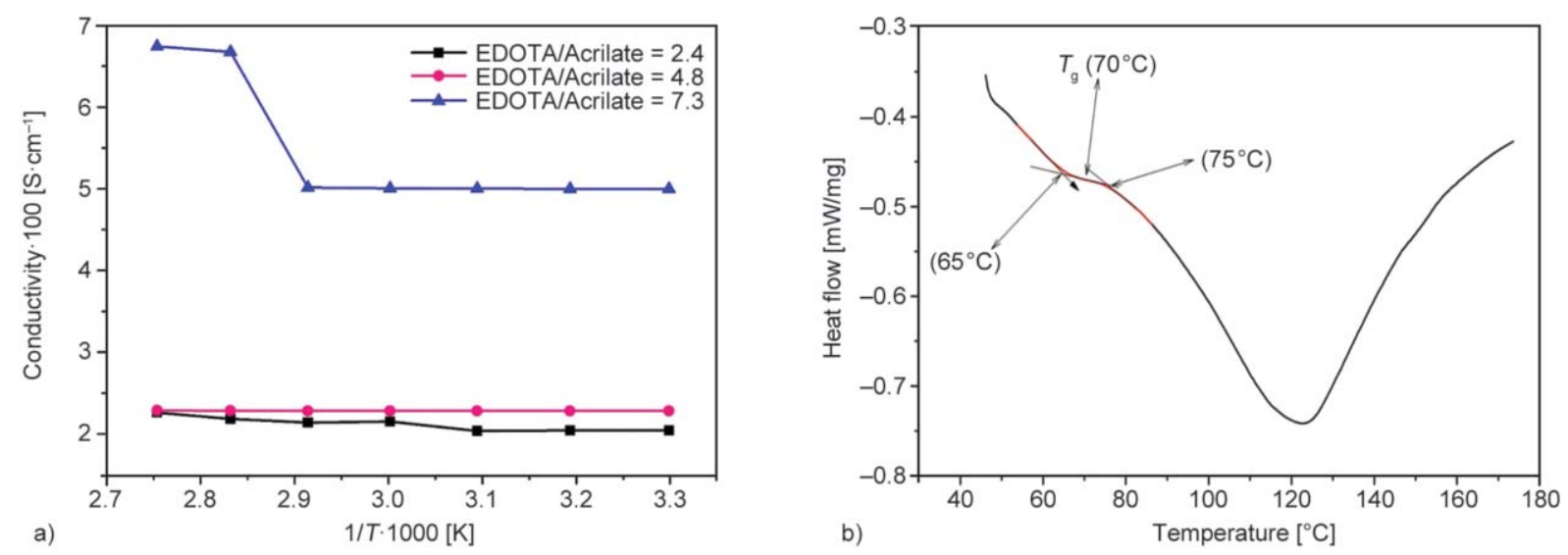

Figure 4. Plot of conductivity against the reciprocal of temperature on the composite films (a) and differential scanning calorimetric experiment of poly(EDOT-co-TCA)-polyacrylate blend film with EDOT/acrylate ratio of 7.3 (b)

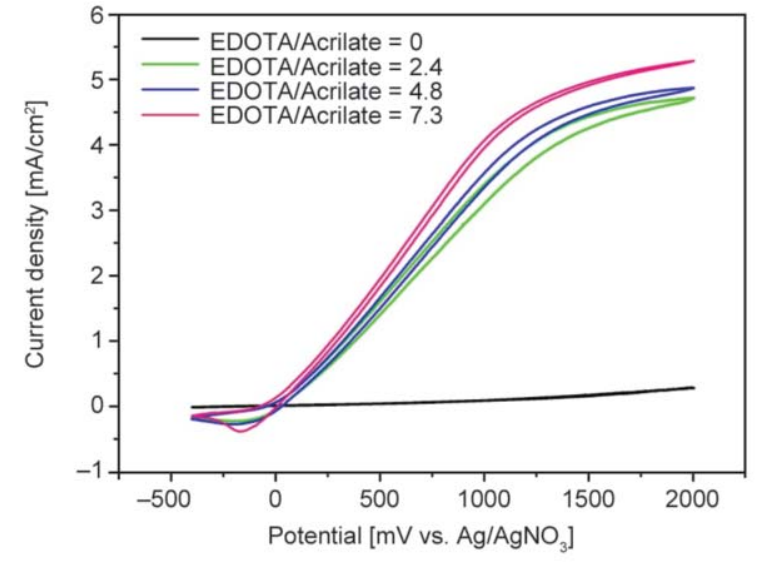

Figure 5. Cyclic voltammograms of poly(EDOT-co-TCA)polyacrylate blend films varying the EDOT/acrylate ratio in an acetonitrile/AcOH $(7 / 1, \mathrm{v} / \mathrm{v})$ solution containing $0.1 \mathrm{M}$ tetrabutylammonium tetrafluoroborate $\left(\mathrm{TBABF}_{4}\right)$ and $0.1 \mathrm{M}$ ferrocene at a scan rate of $50 \mathrm{mV} \cdot \mathrm{s}^{-1}$

\subsection{Electropolymerization of aniline on electroactive poly(EDOT-co-TCA)/ polyacrylate blend film}

Polyaniline derivatives can be electropolymerized by using cyclic voltammetry in aqueous media with the addition of acids as a dopant. Typical CVs of aniline electropolymerization on an electroactive substrate of poly(EDOT-co-TCA)/polyacrylate blend film are depicted in Figure 6a. Refer to cycle 25, two redox couples are observed in these $\mathrm{CV}$ s. In positive sweep, the first redox peak presented at ca. $180 \mathrm{mV}$ is well known as the formation of radical cations (polaronic emeraldine) [20], and the second redox couple at ca. $740 \mathrm{mV}$ is the formation of diradical dications (the resonance structures: bipolaronic pernigraniline and protonated quinonediimine [20]) through the oxidation of PAN deposited on the electrode. The first peak current increased with increasing scan cycles, indicating that the deposited amount of electroactive PAN on the electrode surface increases with increasing scans. This direct proportionality is employed to monitor the rate of AN electropolymerization [23]. Anodic peak currents obtained directly from CVs as a function of cycle number (i.e., the reaction time) and plotted in Figure 6b, revealing that the deposition amount of PAN is proportional to cycle number and autoacceleration behavior exists in the electro-polymerization. Also note that the peak current increases with increasing the mole ratio of EDOT/acrylate in these composite substrates. The rate of PAN deposition also increases with increasing with the mole ratio of EDOT/acrylate. This arises from the increase of surface area (Figure 3 ) and active sites on the electroactive films with increasing EDOT/ acrylate ratio. This result is consistent with the electrical conductivity of these $\mathrm{P}(\mathrm{EDOT}-\mathrm{co}$-TCA)/polyacrylate films and the CVs of the respective $25^{\text {th }}$ cycle PAN deposition in Figure 6c. Figure 6d shows SEM image of PAN-deposited film. During electropolymerization, the acid-doping AN monomers in solution deposited, polymerized, and then converted into the dense film on the substrate through the sequential processes of aggregation, fusion, and stacking.

\subsection{Application of PAN/poly(EDOT-co-TCA)/ polyacrylate film in electrochromic device}

The electrochromic behavior of PEDOT:PSS has been confirmed by other authors $[15,16]$. An electrochromic device $\mathrm{P}(\mathrm{EDOT}-\mathrm{co}-\mathrm{TCA}) /$ polyacrylate $(\sim 8 \mu \mathrm{m}) \quad \| \quad$ LiClO$_{4}$-PEO-PC $(\sim 20 \mu \mathrm{m}) \|$ PAN $(\sim 100 \mathrm{~nm}) \mid \mathrm{P}($ EDOT-co-TCA $) /$ polyacrylate $(\sim 8 \mu \mathrm{m})$ was assembled using a front electrode of PAN 

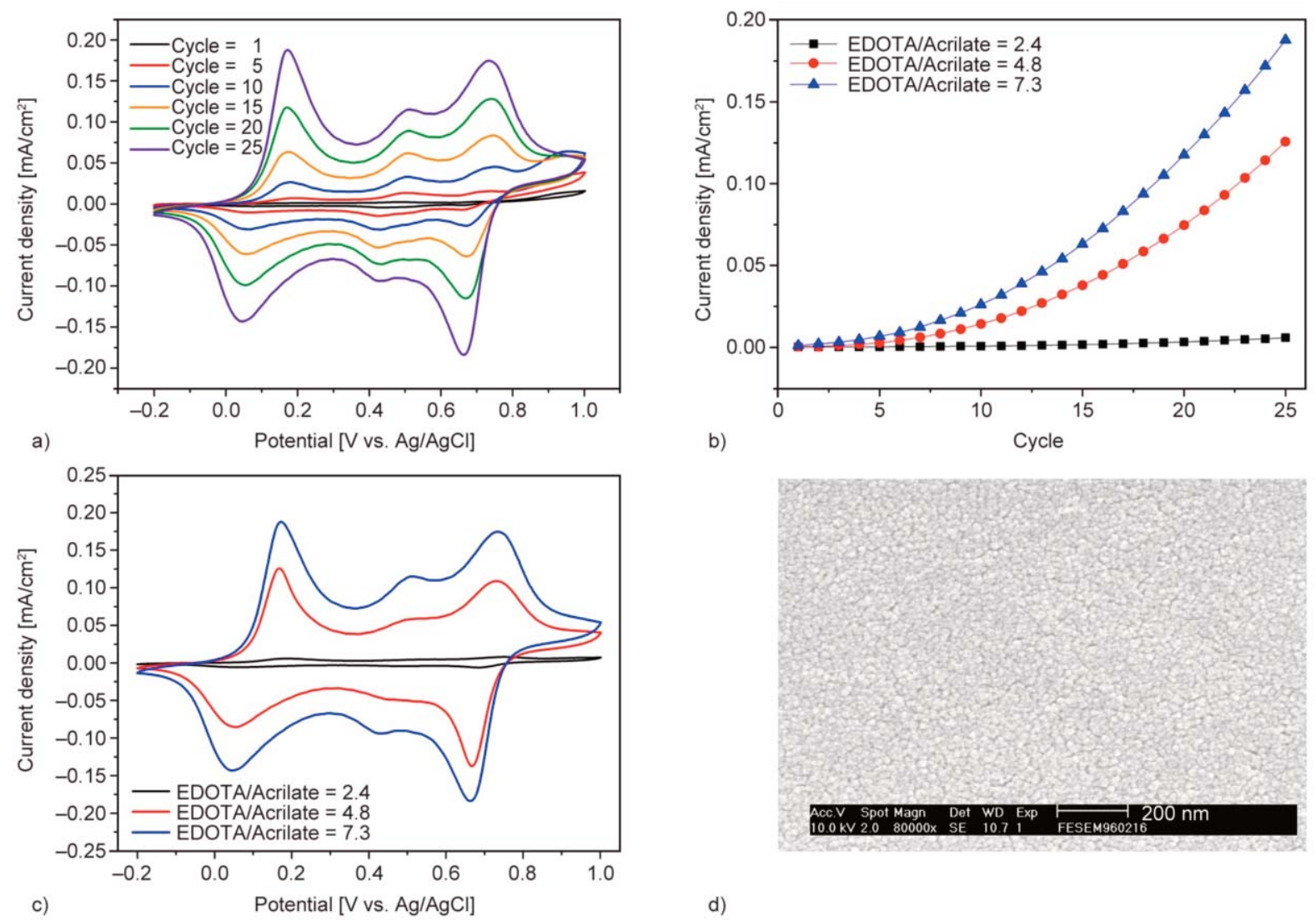

d)

Figure 6. (a) CVs of aniline electro-polymerization on poly(EDOT-co-TCA)-polyacrylate blend film with EDOT/acrylate ratio of 7.3 in $0.1 \mathrm{~N}$ aniline containing $1 \mathrm{~N} \mathrm{HClO}_{4}$ aqueous solution at a scan rate of $50 \mathrm{mV} \cdot \mathrm{s}^{-1}$; (b) plot of $1^{\text {st }}$ peak current density against $\mathrm{CV}$ sweep cycle (reaction time) on the composite films taken from CVs as Figure 6a; (c) CVs of twenty five-cycle deposited PAN on poly(EDOT-co-TCA)-polyacrylate blend film with various EDOT/acrylate ratios; (d) SEM image of PAN-deposited poly(EDOT-co-TCA)-polyacrylate composite film with the EDOT/acrylate ratio of 7.3

$(\sim 100 \mathrm{~nm}) \mid \mathrm{P}($ EDOT-co-TCA $) /$ polyacrylate and a back electrode of $\mathrm{P}(\mathrm{EDOT}-\mathrm{co}-\mathrm{TCA}) /$ polyacrylate. Transmittance spectra recorded at +2.0 and $-2.0 \mathrm{~V}$ yield the highest optical contrast of $36.6 \%$ (at $\lambda_{\max }=$ $645 \mathrm{~nm}$ ) in Figure 7 for this device. This value of optical contrast is higher than that of $\sim 25 \%$ for self-assembling film of polyaniline [2]. The response time of color-bleaching and the stability during consecutive scans in the device were estimated using doublepotential-step chronamperometry. Figure 8 demonstrates current-time and absorbance-time profiles for the device at the first and $200^{\text {th }}$ cycles. The current decay reached zero after $10 \mathrm{~s}$ in the coloring process, while the decay to zero current was only $7 \mathrm{~s}$ in the bleaching process. This result reflects the faster dedoping of $\mathrm{PAN}^{+} \mathrm{ClO}_{4}^{-}$and de-insertion of $\mathrm{Li}^{+}$ion from poly(EDOT-co-TCA). Values of transmittance variation were simultaneously recorded at the wavelength of highest optical contrast. Table 1 summarizes the results obtained for the devices in the first and $200^{\text {th }}$ cycles of double-potential-step spectrochrono-

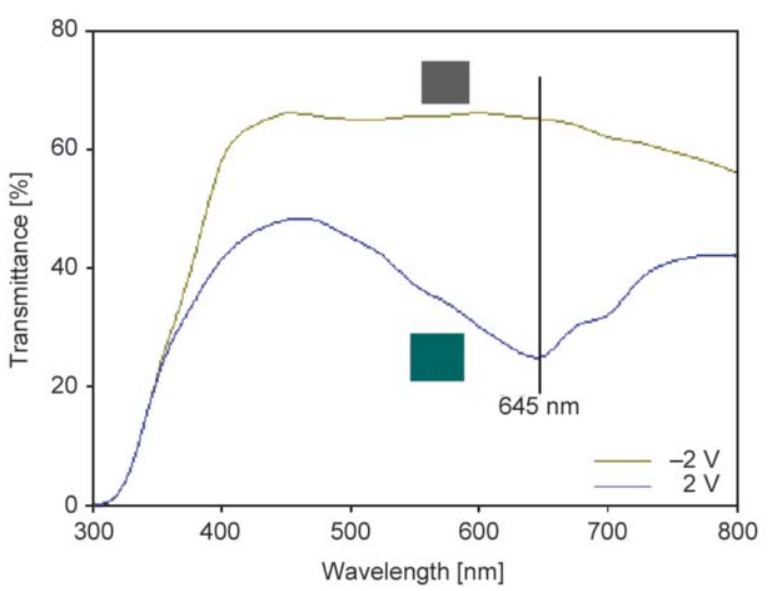

Figure 7. Transmittance spectra of the ECD device using PAN deposited P(EDOT-co-TCA)/polyacrylate film switching potentials between +2.0 and $-2.0 \mathrm{~V}$

amperometry experiments. An examination of Table 1 reveals that the complete device was subjected to 200 charge/discharge cycles and found a similar tendency for this full-polymer-based electrochromic device. The optical contrast $(\Delta T)$ of the device was var- 
(a)

(b)

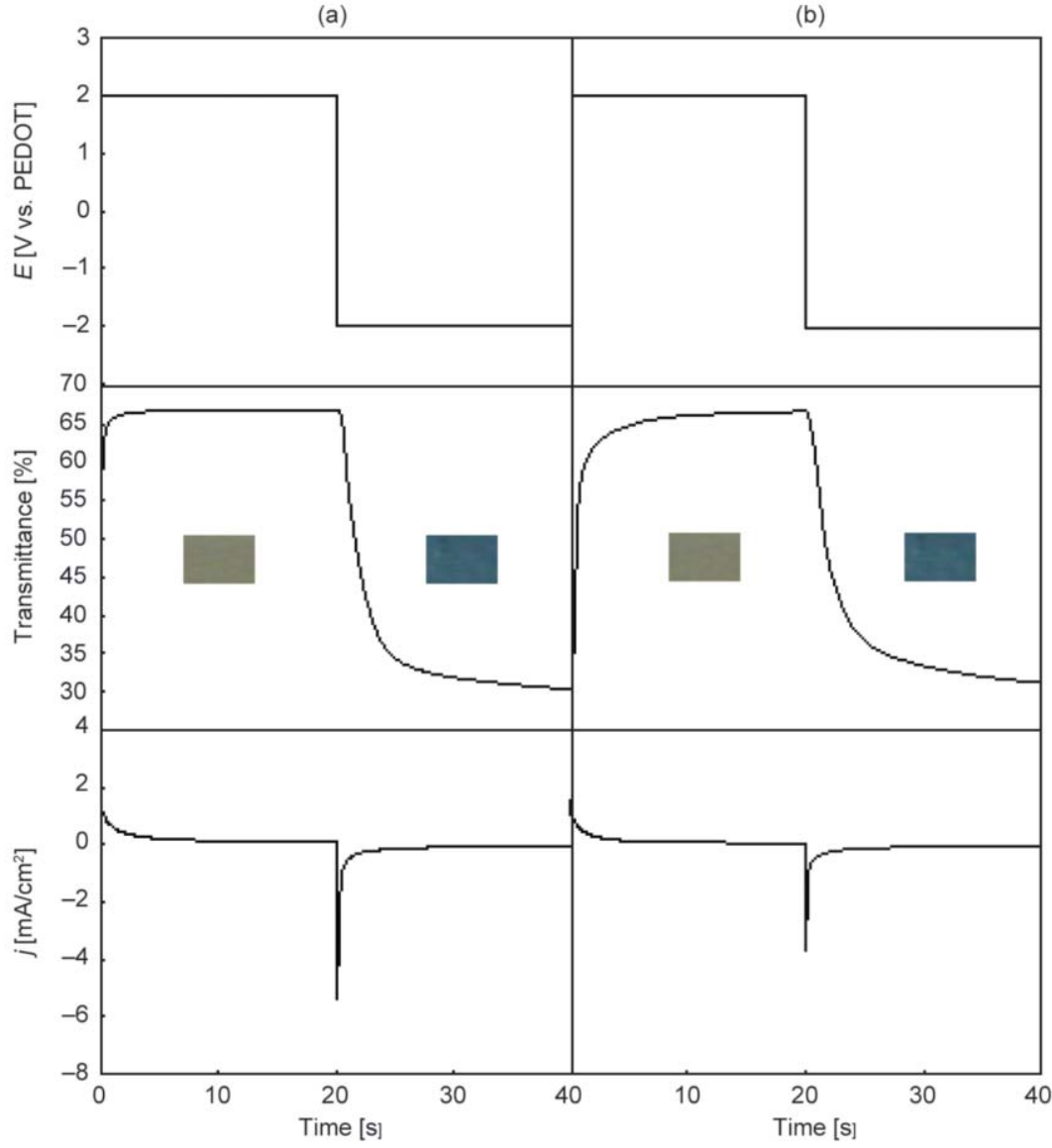

Figure 8. Transmittance variation at $\lambda=645 \mathrm{~nm}$, and current response for the device: $\mathrm{P}($ EDOT-co-TCA)/polyacrylate $\mid$ PAN $(\sim 100 \mathrm{~nm}) \| \mathrm{LiClO}_{4}$-PEO-PC $\| \mathrm{P}($ EDOT-co-TCA $) /$ polyacrylate during double potential step chronoamperometry $\left(E_{1}=+2.0 \mathrm{~V}, E_{2}=-2.0 \mathrm{~V}\right.$ and $\left.t_{\text {step }}=20 \mathrm{~s}\right)$. (a) First and (b) $200^{\text {th }}$ cycle, respectively.

Table 1. Electrochromic properties of the full-polymer device at $\lambda_{\max }$ of $645 \mathrm{~nm}$

\begin{tabular}{|c|c|c|c|c|}
\hline $\begin{array}{c}\text { Number } \\
\text { of step }\end{array}$ & $\begin{array}{c}\Delta \boldsymbol{T} \\
{[\%]}\end{array}$ & $\begin{array}{c}\boldsymbol{\tau} \text { (gray form) } \\
{[\mathbf{s}]}\end{array}$ & $\begin{array}{c}\boldsymbol{\tau} \text { (blue form) } \\
{[\mathbf{s}]}\end{array}$ & $\begin{array}{c}\boldsymbol{\eta} \\
{[\%]}\end{array}$ \\
\hline 1 & 36.6 & 3.1 & 5.9 & 120.5 \\
\hline 200 & 34.9 & 4.9 & 8.1 & 102.5 \\
\hline
\end{tabular}

ied from 36.6 to $34.9 \%$ having the change of the color from gray to blue. From the spectrochronoamperometry experiments, the stability of blue form transmittance is shorter than that of the gray form for PAN on poly(EDOT-co-TCA) film, reflecting a faster response time for the reduction of PAN/oxidation of poly(EDOT-co-TCA) than for the reverse process due to the faster kinetics of dopant ion diffusion. Moreover, the response time is relatively short in the PAN | poly(EDOT-co-TCA)-based device. Also note that the current-time curve was reduced to a certain extent after 200 double-potential steps (refer to Figure 8 ). The Coulombic efficiency $(\eta)$, calculated from the ratio between the coloring and the bleaching charge, was $120.5 \%$ in the first cycle and decreased to $102.5 \%$ in the $200^{\text {th }}$ cycle, indicating that the dop- ing/dedoping process of polymeric film needs a certain number of charge/discharge cycles to stabilize. This is because the conformational changes occur during the redox processes.

\section{Conclusions}

We demonstrated in situ photopolymerization for the cast film involving binary components to prepare a transparent electroactive conductive composite film. The free radicals from acrylate special PIs were able to significantly promote cationic polymerization of thiophenes. The obtained thiophenes and acrylate polymer composite (poly(EDOT-co-TCA)/polyacrylate) provided a basis for preparing functional materials with electrical conductivity and electroactivity, which was potentially useful in the applications of electrochromic devices. The poly(EDOT-co-TCA)/ polyacrylate composite film was used as an electroactive substrate for depositing conducting polyaniline films. This PAN| poly(EDOT-co-TCA) film was employed to fabricate an electrochromic device $\mathrm{P}($ EDOT-co-TCA $) /$ polyacrylate $(\sim 8 \mu \mathrm{m}) \| \mathrm{LiClO}_{4}{ }^{-}$ 
PEO-PC $(\sim 20 \mu \mathrm{m}) \|$ PAN $(\sim 100 \mathrm{~nm}) \mid \mathrm{P}(\mathrm{EDOT}-c o-$ TCA $)$ /polyacrylate $(\sim 8 \mu \mathrm{m})$ and demonstrated coulombic efficiency of $\sim 102 \%$ and optical contrast $(\sim 37 \%)$. The device possesses perceptible color changes from blue to gray with switching potentials from +2.0 to $-2.0 \mathrm{~V}$.

\section{Acknowledgements}

Financial support from the Ministry of Science and Technology in Taiwan (MOST 105-2221-E-390-030 and MOST 1042221-E-390-023) is gratefully acknowledged.

\section{References}

[1] McDiarmid A. G.: Synthetic metals: A novel role for organic polymers. Synthetic Metals, 125, 11-22 (2001). https://doi.org/10.1016/S0379-6779(01)00508-2

[2] Yang C-H., Chih Y-K., Wu W-C., Chen C-H.: Molecular assembly engineering of self-doped polyaniline film for application in electrochromic devices. Electrochemical and Solid-State Letters, 9, C5-C8 (2006). https://doi.org/10.1149/1.2131242

[3] Roncali J.: Synthetic principles for bandgap control in linear $\pi$-conjugated systems. Chemical Reviews, 97, 173-206 (1997). https://doi.org/10.1021/cr950257t

[4] Dizge N., Gunaydin O., Yilmaz F., Tanriseven A.: Immobilization of invertase onto poly(3-methylthienyl methacrylate)/poly(3-thiopheneacetic acid) matrix. Biochemical Engineering Journal, 40, 64-71 (2008). https://doi.org/10.1016/j.bej.2007.11.017

[5] Olmedo L., Hourquebie P., Jousse F.: Microwave absorbing materials based on conducting polymers. Advanced Materials, 3, 373-377 (1993). https://doi.org/10.1002/adma.19930050509

[6] Yagci Y., Yilmaz F., Kiralp S., Toppare L.: Photoinduced polymerization of thiophene using iodonium salt. Macromolecular Chemistry and Physics, 206, 1178 1182 (2005). https://doi.org/10.1002/macp.200500084

[7] Englebienne P., Weiland M.: Synthesis of water-soluble carboxylic and acetic acid-substituted poly(thiophenes) and the application of their photochemical properties in homogeneous competitive immunoassays. Chemical Communications, 14, 1651-1652 (1996). https://doi.org/10.1039/CC9960001651

[8] Iarossi D., Mucci A., Schenetti L., Seeber R., Goldoni F., Affronte M., Nava F.: Polymerization and characterization of 4,4'-bis(alkylsulfanyl)-2,2'-bithiophenes. Macromolecules, 32, 1390-1397 (1999). https://doi.org/10.1021/ma9813340

[9] Chayer M., Faid K., Leclerc M.: Highly conducting water-soluble polythiophene derivatives. Chemistry of Materials, 9, 2902-2905 (1997). https://doi.org/10.1021/cm970238v
[10] Piletsky S. A., Piletska E. V., Karim K., Davis F., Higson S. P. J., Turner A. P. F.: Photochemical polymerization of thiophene derivatives in aqueous solution. Chemical Communications, 2004, 2222-2223 (2004). https://doi.org/10.1039/B408387C

[11] Yang C-H., Huang L-R., Chih Y-K., Chung S-L.: Simultaneous molecular-layer assembly and copolymerization of aniline and o-aminobenzenesulfonic acid for application in electrochromic devices. The Journal of Physical Chemistry C, 111, 3786-3794 (2007). https://doi.org/10.1021/jp065891x

[12] Bella F., Ozzello E. D., Sacco A., Bianco S., Bongiovanni R.: Polymer electrolytes for dye-sensitized solar cells prepared by photopolymerization of PEG-based oligomers. International Journal of Hydrogen Energy, 39, 3036-3045 (2014). https://doi.org/10.1016/j.jhydene.2013.06.110

[13] Bella F., Ozzello E. D., Bianco S., Bongiovanni R.: Photo-polymerization of acrylic/methacrylic gel-polymer electrolyte membranes for dye-sensitized solar cells. Chemical Engineering Journal, 225, 873-879 (2013). https://doi.org/10.1016/j.cej.2013.04.057

[14] Bella F., Leftheriotis G., Griffini G., Syrrokostas G., Turri S., Grätzel M., Gerbaldi C.: A new design paradigm for smart windows: Photocurable polymers for quasi-solid photoelectrochromic devices with excellent long-term stability under real outdoor operating conditions. Advanced Functional Materials, 26, 1127-1137 (2016). https://doi.org/10.1002/adfm.201503762

[15] Radzir N. N. M., Hanifah S. A., Ahmad A., Hassan N. H., Bella F.: Effect of lithium bis(trifluoromethylsulfonyl)imide salt-doped UV-cured glycidyl methacrylate. Journal of Solid State Electrochemistry, 19, 30793085 (2015).

https://doi.org/10.1007/s10008-015-2910-z

[16] Fouassier J. P., Lalevée J.: Photoinitiators for polymer synthesis: Scope, reactivity and efficiency. Wiley-VCH, Weinheim (2012). https://doi.org/10.1002/9783527648245

[17] Tehfe M. A., Louradour F., Lalevée J., Fouassier J-P.: Photopolymerization reactions: On the way to a green and sustainable chemistry. Applied Sciences, 3, 490514 (2013). https://doi.org/10.3390/app3020490

[18] Doyranlı C., Baycan Koyuncu F.: Carbazole based electrochromic polymers with benzoazole units: Effect of heteroatom variation on electrochromic performance. Express Polymer Letters, 10, 762-770 (2016). https://doi.org/10.3144/expresspolymlett.2016.70

[19] Kumar A., Welsh D. M., Morvant M. C., Piroux F., Abboud K. A., Reynolds J. R.: Conducting poly(3,4alkylenedioxythiophene) derivatives as fast electrochromics with high-contrast ratios. Chemistry of Materials, 10, 896-902 (1998). https://doi.org/10.1021/cm9706614 
[20] Jonas F., Heywang G., Gladbach B., Schimdtberg W., Heinze J., Dietrich M.: Method of imparting antistatic properties to a substrate by coating the substrate with a novel polythiophene. U.S. Patent 5035926, USA (1991).

[21] Granqvist C. G., Hultåker A.: Transparent and conducting ITO films: New developments and applications. Thin Solid Films, 411, 1-5 (2002).

https://doi.org/10.1016/S0040-6090(02)00163-3
[22] Sotzing G. A., Reynolds J. R., Steel P. J.: Poly(3,4-ethylenedioxythiophene) (PEDOT) prepared via electrochemical polymerization of EDOT, 2,2'-bis(3,4-ethylenedioxythiophene) (BiEDOT), and their TMS derivatives. Advanced Materials, 9, 795-798 (1997). https://doi.org/10.1002/adma.19970091005

[23] Yang C-H., Yang T-C., Chih Y-K.: Mixture design applied to electrochemical polymerization of ternary aniline derivatives on ITO electrodes. Journal of the Electrochemical Society, 152, E273-E281 (2005). https://doi.org/10.1149/1.1990499 